\title{
La desapropiación corporal: la autopercepción en personas con lesiones permanentes, violencia sexual o tortura crónica
}

\author{
Rafael J. Salín-Pascual* \\ Departamento de Psiquiatría y Salud Mental, Facultad de Medicina, Universidad Nacional Autónoma de México, Ciudad de México, México
}

Sobre el mito de Sísifo «No hay más que un problema filosófico verdaderamente serio: el suicidio. Juzgar si la vida vale o no vale la pena de vivirla es responder a la pregunta fundamental de la filosofía».

\section{Resumen}

Albert Camus, El mito de Sísifo

El dolor físico crónico por enfermedad o tortura produce la pérdida del control sobre el cuerpo en general y de algunas funciones en particular en las personas que lo experimentan. Existe una relación recíproca entre poseer un cuerpo y ser el agente del movimiento y acciones del mismo. En neurofilosofía esto se denomina "sentido de agencia». En situaciones de estrés moderado el cuerpo puede perder el control de las funciones viscerales, pero sigue percibiendo el cuerpo como propio. Si el estrés es intenso, prolongado y con desesperanza, el cuerpo deja de sentirse como propio, se transforma en el enemigo, y esto se conoce como «descorporización». La estimulación magnética transcraneal de la corteza de asociación derecha parietal, occipital y temporal produce fenómenos de agencia y percepción del uno mismo, sitio del fenómeno de la descorporización. Este mismo fenómeno se presenta en personas bajo tortura crónica o violencia sexual prolongada, 0 con enfermedades crónicas dolorosas. Este tipo de dolor colapsa los sistemas cognitivos de primera persona y genera el fenómeno conocido como "sensación de desapropiación corporal» (SDC).

Palabras clave: Sentido de agencia. Estrés postraumático. Descorporización. Autoscopia. Tortura.

\section{Corporal expropriation: self-perception in people with permanent injuries, sexual violence or chronic torture}

\begin{abstract}
Chronic physical pain due to illness or torture, results in the loss of control over the body in general and some functions in particular in human beings who experience them. There is a reciprocal relationship between owning a body and being the
\end{abstract}

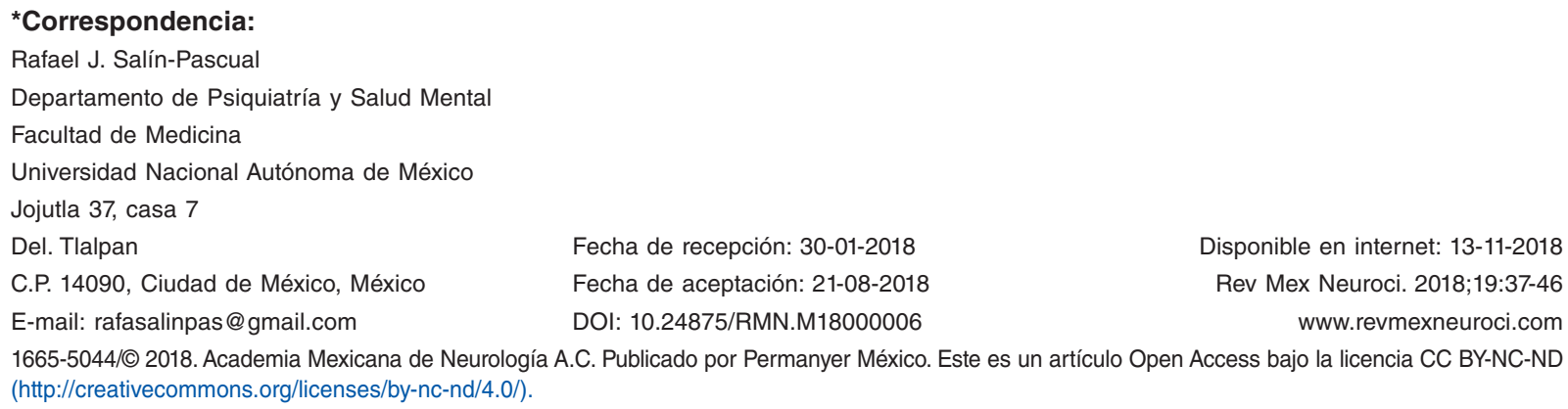

Fecha de recepción: 30-01-2018

Fecha de aceptación: 21-08-2018

DOI: 10.24875/RMN.M18000006

Disponible en internet: 13-11-2018 Rev Mex Neuroci. 2018;19:37-46 www.revmexneuroci.com (http://creativecommons.org/licenses/by-nc-nd/4.0/). 
Rev Mex Neuroci. 2018;19

agent of movement and actions of that body. This in neurophilosophy is called "sense of agency." In situations of moderate stress the body can lose control of visceral functions, but still perceives the body as its own. If the stress is intense, prolonged and hopeless, the body stops feeling like its own, it becomes the enemy, and this is known as disembodiment. The transcraneal magnetic stimulation of the right parietal, occipital and temporal association cortex produces the descorporization phenomena, in which the person perceives the phenomenon of not to recognize their body as the agency of movement and sensation. This same phenomenon occurs in people under chronic torture, prolonged sexual violence, and chronic painful diseases. These types of pain collapse the cognitive systems of the first person and generate the phenomenon known as "Sensation of corporal desapropiation".

Key words: Sense of agency. Postraumatic Stress Disorder. Descorporization. Autoscopy. Torture.

\section{Introducción}

La experiencia vivencial de los sobrevivientes a torturas o eventos traumáticos ha desarrollado una serie de reportes clínicos en los que lo más frecuente es referir que se ha perdido la sensación de control sobre el propio cuerpo ${ }^{1}$. Esto es el sentido de agencia que se tiene sobre la corporeidad o poseer un cuerpo. De acuerdo con estos reportes, existe una continuidad recíproca entre ser el poseedor de un cuerpo y el sentido de ser el agente que lo controla, o sentido de agencia (sense of agency, en inglés), que es tener la capacidad para percibir y ejecutar acciones y las consecuencias que devengan de ellas ${ }^{2}$. En situaciones de estrés extremo, las personas mantienen el control sobre su propio cuerpo, como parte de «sí mismos", aun cuando pierdan el control sobre sus funciones. Por ejemplo, las personas pueden perder la inhibición sobre funciones autonómicas como defecar, orinar, vomitar, que están fuera de su control consciente habitual, pero siguen percibiendo el cuerpo como propio y con dolor, que les informa de las zonas de lesión ${ }^{3}$.

La percepción de nuestro cuerpo y el identificarnos con éste es una condición que pocas veces se cuestiona, porque se da como una de esas situaciones que David Hume, padre del empirismo inglés, refirió como constantes universales, que, sin embargo, pueden alterarse ${ }^{4}$. Por ejemplo, si una persona escucha la voz de un familiar sin mirarlo, espera que al ponerse en su campo visual vea a esa persona que tiene ese tipo de voz, que camina con esa cadencia o que llega a determinada hora a un sitio acordado. En el caso de que esto no sea así, la reacción universal es la de asombro. En un experimento, se colocaron niños menores de un año delante de un teatro guiñol en miniatura. Los infantes veían cómo se colocaba una pelota en una corredera, y luego otra, que resbalaban hacia el centro del telón. Cuando éste se abría, lo lógico sería ver dos pelotas. Si esto sucedía, los niños lo celebraban con risas; pero si sólo veían una bola, cuando en realidad habían visto dos bolas resbalándose, sus rostros mostraban asombro, pues se rompía una constate universal.

\section{Localización cerebral del «uno mismo»}

En nuestra corteza cerebral, en las zonas somatosensoriales y motoras (áreas 3, 1, 2 y 4 de Broadman), se tiene la localización del cuerpo como un equivalente a un mapa de brazos, piernas, dedos, lengua, etc. ${ }^{5}$. Este mapa es desproporcionado, pues las manos y el dedo pulgar, junto con la lengua y la orofaringe, son muy grandes, mientras que los genitales son minúsculos y la espalda casi no existe. Este mapa, una especie de «GPS corporal», reacciona si el neurocirujano, por ejemplo, estimula cada una de esas regiones en una cirugía o en la estimulación magnética transcraneal cuando se busca el área motora. Ahí la persona, al encontrar el umbral, moverá los dedos o la mano contralaterales al sitio de colocación del magneto. Pero esto nada tiene que ver con nuestra autopercepción, la cual se almacena en una región de asociación cortical, la parietotemporooccipital derecha ${ }^{6}$. Nuestra autoimagen o la identidad de uno mismo es un constructo (como la mayoría de lo que creemos que es la realidad), y se construye desde los estadios prenatales, cuando el feto, utilizando el sentido del tacto, se delimita, y continuará a lo largo de la gestación y en el periodo posnatal, con algunos puntos culminantes, como la identificación de nuestra imagen en el espejo y la integración de nuestra corporeidad en el contexto biológico-genérico y social. Los fenómenos clínicos como el miembro fantasma, la anosognosia y la asomatognosia son ejemplos extremos de cómo la agencia corporal puede estar comprometida, igual que en la condición de las personas transexuales, en las que, sin dejar de reconocer que el cuerpo que poseen es el suyo, existe una incongruencia con la sensación de género respecto al fenotipo de éste ${ }^{7,8}$. La estimulación magnética transcraneal en el área parietotemporooccipital derecha 
proporciona la sensación de extracorporeidad y en algunos casos de autoscopia, es decir, la de verse o percibirse fuera del propio cuerpo. Esto nos permite, a modo de premisa, argumentar que el desacoplamiento de la corporeidad es posible y tiene una función evolutiva, quizás de protección ante eventos catastróficos ${ }^{6}$.

\section{Sensación de desapropiación corporal}

En condiciones de estrés prolongado, como sucede con los prisioneros de guerra, secuestrados, violencia sexual o en personas con padecimientos crónicos dolorosos, el sistema cognitivo se colapsa y las personas pueden tener la ilusión de que no poseen su cuerpo, en parte o por completo ${ }^{9-13}$. Esto se conoce como "sensación de desapropiación corporal» (SDC) y se caracteriza por:

- Pérdida de los recursos físicos, cognitivos, y emocionales para poder hacer frente a la situación que los ha colocado en esa desapropiación corporal.

- Percepción de que todos los objetos que rodean a la víctima, incluyendo su propio cuerpo, son hostiles.

- Existe una percepción de tortura constante.

- Hay una sensación severa de desesperanza.

- Aceptación de una creencia de aniquilación inminen-

te, que además es deseada por quien sufre, como salida de esa experiencia ${ }^{14}$.

Los especialistas que estudian este tipo de problemas han observado que hay tres condiciones en la clínica psiquiátrica que pueden dar como resultado la SDC:

- Conductas de lesión corporal autoafligida (self harming behavior [SHB]).

- Estrés postraumático disociativo.

- Formas complejas de estrés postraumático.

Estos tres fenómenos no están tan separados o diferenciados como se pensaba, pues tienen como factores comunes la exposición crónica y repetida a eventos traumáticos, en particular a los infligidos por otros seres humanos, como son los secuestros, abusos sexuales, abusos físicos, prisioneros políticos, etc. $^{14}$. Es importante aclarar que no todas las personas sometidas a los eventos antes mencionados desarrollan síndrome de estrés postraumático (SEPT), sino que se requieren otros factores, que se están estudiando en la actualidad y que podrían ser el equivalente de una vulnerabilidad genética ${ }^{10}$. En algunos casos sólo hay una desapropiación corporal a ciertas regiones, aunque es cierto que otras personas desarrollan la SDC completa, y que no existe una correlación respecto a la intensidad, frecuencia o cronicidad de los estímulos traumáticos. Factores como la edad de inicio, las condiciones psiquiátricas preexistentes, la personalidad pretraumática, los estilos de afrontamiento, entre otros, pueden explicar las diferencias entre repercusiones parciales o generalizadas sobre el sentido de agencia corporal ${ }^{15}$.

El trauma severo y prolongado se ha denominado SEPT de tipo II. Hay varios estudios que han encontrado que las conductas, pensamientos e intentos suicidas están relacionados con la exposición a un trauma de tipo II. Las conductas Ilamadas de manera global como suicidas se incrementan con la severidad de los estresores y la frecuencia de los traumas. Sin embargo, no hay una explicación completa de este fenómeno. Se han propuesto factores como sensación de culpa, vergüenza, sensación de desesperanza o incapacidad para imaginarse una situación liberadora en el futuro ${ }^{15-17}$.

Una alternativa de explicación a ello es que el intento de suicidio es, en sí mismo, una lesión corporal autoinfligida. Así, las conductas de autolesión o SHB podrían estar contenidas en el mismo espectro o continuidad de lesión al cuerpo infligida por el poseedor del mismo. Para algunos autores el SHB funciona como un regulador del estado de ánimo: intentos suicidas, cortarse y otras conductas de autolesión sirven como reguladores del estado de ánimo, sobre todo en lo referente a los estados difusos de ansiedad ${ }^{18,19}$.

La experiencia de SHB incluye: cortarse, quemarse, administrase latigazos (flagelarse), golpearse en regiones del cuerpo, pellizcarse severamente, ruptura de huesos, aplicación de aparatos de tortura en el cuerpo crónicamente, como son los cilicios y otros accesorios para provocar dolor de manera deliberada ${ }^{20-23}$. Algunas de estas prácticas se han asociado a experiencias tempranas de abuso sexual, sobre todo en mujeres, a quienes se violentaron desde la infancia. El SHB suele aparecer a los 12-14 años de edad y es mucho más frecuente en niñas. Cuando ocurre en niños o adolescentes, puede tener una connotación de iniciación para pertenecer a una banda o grupo juvenil, como los emos, góticos, darketos, etc. En EE.UU. se atienden a 100,000 adolescentes anualmente por este tipo de autolesiones ${ }^{24}$. Los adolescentes refieren cortarse o lesionarse para detener los «malos pensamientos» 0 para «sentir algo, aunque sea dolor». Los sitios más comunes de los cortes son las muñecas, los antebrazos, los tobillos, los muslos.; en general, áreas que pueden cubrirse para que no las detecten los padres o cuidadores. Los reportes de los jóvenes que se cortan suelen indicar que tienen la sensación de estar 
«entumecidos», «no sentir nada» 0 «sentirse muertos». Niegan el dolor en el momento de cortarse, aunque en las primeras ocasiones que se hacen los cortes sí hay dolor. Algo similar se observa con la tricotilomanía, en donde al arrancarse los cabellos ya no se experimenta dolor, pero sí una sensación neutra o ansiolítica ${ }^{25}$.

En la China existía una sentencia a muerte que consistía en cortar al condenado en mil pedazos para finalmente decapitarlo. Para ello se le administraban grandes cantidades de opio, con lo que las imágenes que se tienen de ese martirio muestran rostros felices, a pesar de los cientos de cortes y mutilaciones. Salvador Elizondo, un escritor mexicano, escribió una novela corta llamada Farabeuf o la crónica de un instante en la que describe en un relato, ubicado en la China, este tipo de sacrificio, denominado Ling Ching o Leng T'ché, que en español significa 'muerte por mil cortes' $^{26,27}$.

En la actual clasificación psiquiátrica de la American Psychiatric Association, el llamado DSM-5, se ha separado el estrés postraumático de los trastornos por ansiedad, en un grupo de entidades separadas. Éstas se denominan «alteraciones por trauma y otras relacionadas a eventos estresantes» (Tablas 1-3) ${ }^{28}$.

En el subtipo SEPT disociativo (SEPT-D) los estados de disociación son relativamente más elevados que el resto de los síntomas que acompañan al SEPT. Estos síntomas incluyen la despersonalización, la experiencia de estar fuera del observador o estar fuera de uno mismo («esto no me está ocurriendo a mí» 0 «esto es como un sueño»). En un estudio con veteranos de guerra de EE.UU., reportado en el año 2012, se encontró que el $6 \%$ de una muestra de estos pacientes con SEPT presentaban un predominio de síntomas disociativos. Una característica común en estas personas es que la mayoría tenían antecedentes de traumas de naturaleza sexual ${ }^{29}$.

En la forma del SEPT complejo (SEPT-C) hay el antecedente de alteraciones completas en cuanto a los criterios diagnósticos, pero se agregan otros, como dificultades en la regulación emocional, en sus capacidades de vinculación interpersonal, alteraciones en sus esquemas de creencias previas, y problemas en la regulación y organización somáticos. Estas personas tienen traumas prolongados, como resultado de los cuales sienten la necesidad de desarrollar unas formas diferentes de realidad para sobrevivir. El ejemplo que se cita en este contexto es el de los prisioneros de guerra o políticos.
Tabla 1. Trastornos relacionados por trauma y factores de estrés según el DSM-5

Trastorno de apego reactivo

Trastorno de relación social desinhibida

Trastorno por estrés postraumático

Trastorno por estrés agudo

Trastorno de adaptación

Otro trastorno relacionado con trauma y factores de estrés específico

Trastorno relacionado con traumas y factores de estrés no especificados

Ante un trauma severo y prolongado, los sobrevivientes pueden desarrollar formas mixtas de SHB, SEPT-D y SEPT-C. Aunque la descripción de estas manifestaciones es amplia y clara, la pregunta central es: ¿por qué no todas las personas sometidas a situaciones similares desarrollan estas manifestaciones?, la cual aún es motivo de debate. Una hipótesis que se propone es la facilidad que tienen algunas personas para presentar la descorporización, esto es, para poder percibirse como una entidad diferente y separada, lo cual puede ser un factor que les facilite el tener SHB, SEPT-D y SEPT-C. Según esta hipótesis, ante este tipo de estresores extremos estas personas desarrollan una percepción del mundo y de su propio cuerpo como los enemigos a vencer. El hecho de que su cuerpo sea la fuente del dolor, causado por agentes externos o internos, en el caso de las enfermedades, les facilita poder tener manifestaciones de descorporización o desapropiación corporal ${ }^{14}$.

\section{Desapropiación corporal parcial o completa}

Es una sensación de que ciertas partes del cuerpo son extrañas a la persona que las posee. En este sentido, hay tres posibilidades:

- Percibir una extremidad como extraña, pero aún se tiene la sensación de poseerla (síndrome de mano anárquica).

- La extremidad es extraña y no se percibe como propia, sino que pertenece a otra persona (somatofrenia).

- Percibir una extremidad en el cuerpo pero no sentirla como propia (trastorno de identidad de la integridad corporal [body integrity identity disorder, BIID] $)^{8,9,30}$. 
Tabla 2. Trastorno por estrés postraumático (309.81, DSM-5)
A. Exposición a la muerte, lesión grave o violencia sexual, ya sea real o amenaza, en una o más de las siguientes formas:
- Experiencia directa del suceso (s) traumático (s)
- Presencia directa del suceso (s) ocurrido (s) a otros
- Conocimiento de que el suceso (s) traumático (s) ha ocurrido a un familiar próximo o a un amigo íntimo
- Exposición repetida o extrema a detalles repulsivos de suceso (s) traumático (s)
B. Presencia de uno (o más) de los síntomas de intrusión siguientes asociados al suceso (s) traumático (s):
- Recuerdos angustiosos recurrentes, involuntarios e intrusivos
- Sueños angustiosos recurrentes con contenido relativo al evento
- Reacciones disociativas en que se repite o reactúa el evento
- Malestar psicológico intenso o prologado a factores internos o externos que simbolizan o se parecen a un aspecto del evento
- Reacciones fisiológicas intensas a factores internos o externos que simbolizan o se parecen al suceso traumático

C. Evitación persistente de estímulos asociados a los sucesos traumáticos:

- Evitación o esfuerzos para evitar recuerdos o pensamientos angustiosos vinculados al evento traumático

- Evitación o esfuerzo para evitar recordatorios externos que recuerden el evento

D. Alteraciones cognitivas negativas y del estado de ánimo asociadas al suceso traumático:

- Incapacidad para recordar un aspecto importante del suceso traumático

- Creencias o expectativas negativas persistentes y exageradas sobre uno mismo, los demás y el mundo

- Percepción distorsionada persistente de las causas o las consecuencias del suceso

- Estado emocional negativo persistente

- Disminución importante del interés o la participación de actividades significativas

- Sentimientos de desapego o extrañamiento de los demás

- Incapacidad para experimentar emociones positivas

E. Alteraciones en el mantenimiento de alerta y reactividad asociada al suceso traumático, que comienza o empeora después del suceso traumático. Que se caracteriza por al menos dos de los siguientes eventos:

- Comportamiento irritable y arrebatos de furia

- Comportamiento imprudente 0 autodestructivo

- Hipervigilancia

- Respuesta de sobresalto exagerada

- Problemas de concentración

- Alteraciones del sueño

F. La duración de las alteraciones (criterios B, C, D y E) es superior a un mes

G. Las alteraciones causan malestar clínicamente significativo o deterioro social, laboral o en otras áreas significativas

H. La alteración no está relacionada a problemas médicos o uso de sustancias

El síndrome de la mano extraña, síndrome de la mano ajena, síndrome de la mano alienígena o síndrome del Dr. Strangelove, es un trastorno neurológico poco frecuente que se caracteriza por la presencia de movimientos involuntarios e incontrolables de uno de los miembros superiores, además de una sensación de «personificación» o al menos de extrañeza con relación al mismo ${ }^{31}$.

Descrito por primera vez en 1908 por el médico alemán K. Goldstein, el síndrome es común en pacientes sometidos a una comisurotomía (sección del cuerpo calloso que separa ambos hemisferios cerebrales). También puede ocurrir tras neurocirugías en personas que han sufrido accidentes cerebrovasculares, infecciones, neoplasias, aneurismas o en pacientes con enfermedades neurodegenerativas como la enfermedad de Alzheimer o la de Creutzfeldt-Jakob ${ }^{32}$.
En una escena de la película Dr. Strangelove (Dr. Strangelove or: How I Learned to Stop Worrying and Love the Bomb), dirigida por Stanley Kubrick, la mano del personaje doctor Strangelove, interpretado por Peter Sellers, parece cobrar vida propia. En otra película, ésta de Alfred Hitchcock, Marnie (1964), una joven ladrona (Tippi Hedren), pretende robar una suma importante de la caja fuerte del hombre del cual está enamorada (Sean Connery). Ella no puede controlar la mano que intenta robar, aunque se esfuerza por impedirlo. Por otro lado, las somatofrenias corresponden a las anosognosias. Éstas son alteraciones en las que el paciente parece ignorar por completo la existencia de la parálisis en una de sus extremidades. El paciente puede incluso expresar que es capaz de mover el miembro paralizado ${ }^{32}$.

El BIID es una enfermedad psiquiátrica que provoca en el individuo afectado un irresistible deseo por 
Tabla 3. Trastorno de estrés agudo (308.3, DSM-5)

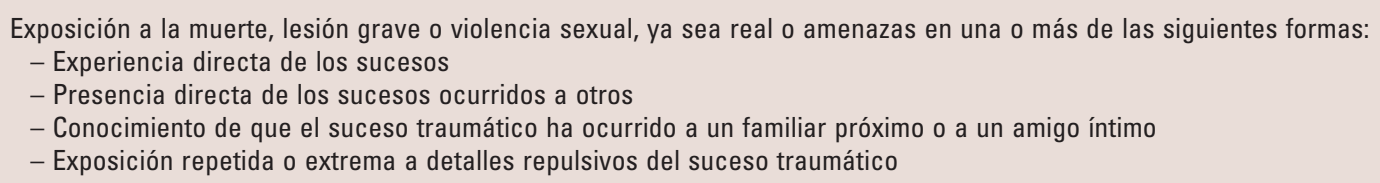

Presencia de nueve o más de los siguientes síntomas de algunas de las categorías de intrusión, estado de ánimo, disociación, evitación y alerta, que comienzan y empeoran después del suceso traumático

Síntomas de intrusión:

- Recuerdos angustiosos recurrentes, involuntarios e intrusivos del suceso

- Sueños angustiosos con temática relacionada con el suceso

- Reacción disociativa

- Malestar psicológico intenso y prolongado

Estado de ánimo negativo:

- Incapacidad para experimentar emociones positivas (felicidad, satisfacción, sentimientos amorosos)

Síntomas disociativos:

- Sentido alterado de la realidad, del entorno de uno mismo sin perder el juicio de realidad

- Incapacidad para recordar aspectos importantes de los sucesos traumáticos

Síntomas de evitación:

- Esfuerzos para evitar recuerdos, pensamientos o sentimientos angustiosos acerca del evento

- Esfuerzos para evitar recuerdos externos, conversaciones o noticias relacionados con el evento

Síntomas de alerta:

- Alteraciones del sueño (insomnio y fragmentación del mismo)

- Irritabilidad y furia

- Hipervigilancia

- Problemas de concentración

- Respuesta exagerada de sobresalto

amputarse una o más extremidades sanas del cuerpo. Los nombres más recientes de este trastorno han reemplazado al de apotemnofilia, debido a la creciente convicción de que esta enfermedad no es una parafilia (v. g., con un tipo de contenido sexual) ${ }^{33}$.

Una persona que sufre de BIID desea tener amputadas una o más de sus extremidades. Este trastorno no debería confundirse con la acrotomofilia, que es el deseo sexual por alguien que ya tiene algún miembro amputado. Dentro de la comunidad BIID, los acrotomófilos son llamados «devotos». De todos modos, parece haber alguna relación entre los dos desórdenes, pues hay individuos que padecen ambos ${ }^{33}$.

Aunque la definición oficial del BIID precisa que es sólo el deseo de la amputación, First, et al. consideran que la enfermedad podría incluir la necesidad de otros impedimentos, como la paraplejía ${ }^{34}$. Evidencias anecdóticas demuestran que un gran porcentaje de personas con BIID necesitan sufrir de distintas discapacidades. Para confirmar esto, First comenzó un estudio en abril de 2007 como seguimiento a su investigación realizada para la quinta edición del Manual diagnóstico y estadístico de los trastornos mentales. Si se descubre que la principal motivación de los pacientes con
BIID es tener alguna discapacidad, este trastorno podría considerarse como una forma del síndrome de Münchhausen. Los enfermos con BIID se perciben incompletos con sus cuatro extremidades y se sienten aliviados tras la amputación. Saben exactamente qué parte de qué miembro debe ser cercenada para calmar su sufrimiento. La solicitud más común es la amputación sobre la rodilla de la pierna izquierda. Los enfermos describen una profunda envidia hacia las personas amputadas y perciben los síntomas antes mencionados como extraños y anormales. Se sienten solos con estos pensamientos y creen que nadie puede entender sus impulsos ${ }^{34}$.

\section{Autoscopia o percepción extracorpórea}

En situaciones de estrés extremo, se sabe que, ante la incapacidad de huir o efectuar un ataque, el sujeto sufre un «congelamiento». Éste es un mecanismo disociativo como mecanismo de adaptación. En este proceso, una parte de la sensación de pertenencia corporal es débil o incluso desaparece de manera intermitente ${ }^{15}$. Esta SDC no es un fenómeno de todo o nada, es decir, no es dicotómica, pues se ajusta a las 
condiciones del medio ambiente. En las experiencias místico-religiosas, la sensación de pertenencia del cuerpo se vuelve débil y aparecen fenómenos como la experiencia de verse afuera del propio cuerpo o autoscopia $^{15}$.

El hecho de que la víctima no se desconecte por completo es una premisa fundamental, pues le va a permitir oscilar entre los estados de desconexión y de percatarse de su medio, para poder activar los mecanismos de ataque o huida en el momento en que esto sea pertinente. En los casos de trauma de tipo II, en los que una persona sufre de violencia, privación de alimentos, tortura, aislamiento, privación del sueño, humillación constante, entre otros insultos, el sistema cognitivo puede colapsarse y no funcionar de manera adecuada $^{9,35,36}$. En condiciones extremas, el sistema de oscilación o negociación entre congelamiento y activación llega a paralizarse, es decir, a anularse por completo, y surge la sensación de desposesión completa de todo el cuerpo ${ }^{13}$.

Y sus efectos son la despersonalización y la desrealización, las cuales, desde el punto de vista fenomenológico, son dos situaciones completamente diferentes. En la despersonalización hay una percepción de tipo onírico; es decir, hay una apreciación como de estar entre neblina y aislado del medio ambiente que rodea a la persona. Aunque la desrealización se asocia a una falta de sensación de poseer un cuerpo, en realidad no es equivalente a la percepción de estar fuera del propio cuerpo (out of the body experience [OBE]) ${ }^{37}$.

La experiencia de verse fuera del cuerpo, ya sea durante el sueño o estando despierto, es una experiencia inquietante, pero al mismo tiempo aleccionadora. La propuesta que se hace es que nuestra percepción de cómo somos en cuanto a lo físico es totalmente una función cerebral, es decir, somos, nos vemos y percibimos con un estado de sesgo o de prejuicio. La autoimagen que tenemos de nosotros y de los demás no se corresponde con lo que está en el exterior ${ }^{6}$. Blanke, et al. ${ }^{6}$ describieron que la experiencia de cercanía a la muerte, como acompañada por autoscopia, es similar a la que se observa en el sueño sueño de movimientos oculares rápidos (MOR). Su revisión se titula: "¿La experiencia de cercanía a la muerte se explica por los sistemas de alerta?".

La autoscopia es una percepción cuyo objeto somos nosotros mismos, es casi una alucinación; lo que ocurre es que nos alucinamos de maneras muy diversas ${ }^{38}$.

La experiencia de verse fuera del cuerpo (saber que somos nosotros mismos y al mismo tiempo percibirse como un espectador) es lo que se conoce como «autoscopia». En el sueño hay por lo menos dos tipos de estos fenómenos: durante el sueño MOR y fuera de éste. En el caso del sueño MOR, la autoscopia es integrada dentro del campo de lo que se está soñando ${ }^{39}$. Fuera del sueño MOR es una experiencia extraña, pero con una narrativa equivalente al pensamiento diurno, es decir, cuando se está despierto.

La experiencia de verse a uno mismo es una experiencia muy frecuente en la mitología, las tradiciones populares y en experiencias espirituales. Una de las funciones en las cuales se reportan con más frecuencia las autocopias es al dormir. La experiencia en este sentido es anecdótica y no existe un método confiable que permita su estudio. Por ejemplo, es común en la parasomnia, conocida como "parálisis del sueño», en donde el que sueña ve parte de su cuerpo y trata de moverlo, situación que produce ansiedad en el durmiente ${ }^{40}$.

La autoscopia ha sido estudiada más como un fenómeno parapsicológico que como la suma de los mapas que el cerebro tiene de uno mismo. Por ejemplo, «la experiencia de cercanía de muerte" y fenómenos de experiencias de "estar fuera del cuerpo" se han connotado como fenómenos extraños y vinculados a una serie de alteraciones neuropsiquiátricas, como la epilepsia, la narcolepsia y en algunas formas de psicosis. En la narcolepsia, gran parte de los fenómenos alucinatorios tan vívidos, llamados «alucinaciones hipnapómpicas e hipnagógicas», son situaciones en las que la persona se visualiza intentando avisar a la persona que duerme a un lado de lo que le sucede para que pueda ser despertada ${ }^{41,42}$.

Sin embargo, todas las noches tenemos fenómenos de autoscopia durante las ensoñaciones, esto es, ya sea que nos vemos como nosotros mismos o como otras personas que asumimos que somos nosotros, 0 incluso en forma de animales. En este contexto no nos percatamos ni tampoco hay alarma, pero es una experiencia cotidiana. Si una función de la actividad onírica es recrear realidades virtuales, ciertamente en ellos somos los protagonistas ${ }^{41}$.

La experiencia más angustiante es la autoscopia en la parálisis del sueño, situación que se presenta como parte de la narcolepsia y de manera aislada en personas sanas. Nuevamente, la experiencia es mucho más frecuente en las personas que se acuestan después de las dos o tres de la madrugada, en donde las latencias a dormir primero y luego al inicio del sueño MOR hacen que se tenga la vivencia de la parálisis, que corresponde a la atonía muscular que se tiene en el sueño MOR, más el componente alucinatorio ${ }^{40}$. Tener sueño delta permite bajar la intensidad de la vivencia 
de lo que se sueña; si por el contrario se tiene sueño MOR de una latencia muy corta, por ejemplo $10 \mathrm{~min}$ después de haberse quedado dormido, la vivencia es muy intensa, tanto que se puede estar totalmente convencido de que lo soñado es real ${ }^{43}$. La autoscopia en esos casos es parcial; uno ve cómo su brazo se estira buscando a la pareja y además piensa que le va a pedir que le sacuda, que le despierte.

Los estudiosos de la OBE proponen que puede haber tres tipos de esta experiencia ${ }^{44}$ :

- Alucinación de tipo autoscopia, en donde las personas perciben un doble de sí mismas pero sin tener la experiencia de la descorporización. Esto es, se ven fuera de su cuerpo, pero siguen percibiéndose como espectadores de eso desde dentro de su propio cuerpo (es como verse en un espejo).

- Heautoscopia, en donde la persona puede ver partes de sus órganos internos o la carencia de éstos. Ambos fenómenos son alucinatorios, pero centrados en los componentes del cuerpo.

- Experiencia OBE, en donde las personas se perciben despiertas pero su centro de conciencia está fuera del cuerpo. Es frecuente que se perciba desde una posición elevada con respecto al cuerpo al cual parecen observar y reflexionar sobre su situación anormal. Esta experiencia se ha reportado en parálisis del sueño y en estados de resucitación de individuos que han tenido suspensión de signos vitales por algunos segundos. Al interrogarles con respecto a esta experiencia, las personas dicen haberse visto desde el techo de la habitación, pero nunca pueden realizar una descripción exhaustiva del número de resucitadores, colores, equipos para resucitar, etc. Se ha propuesto que la resucitación con estimulación extrema, táctil, dolorosa e incluso de las vías vestibulares es la que puede formar parte de este fenómeno.

Este último fenómeno es crítico para poder explicar por qué uno de los mecanismos de defensa ante el estrés extremo es la disociación corporal. Esto ya se refirió en la primera guerra mundial como «neurosis de guerra», pues se encontraron soldados vagando por zonas llamadas «tierra de nadie», los cuales fueron con frecuencia calificados como cobardes, procesados a menudo por cobardía y terminaron sus días en el pelotón de fusilamiento ${ }^{45}$.

La experiencia del propio cuerpo es un proceso fundamental que nos permite interactuar con el mundo exterior. La experiencia del cuerpo se basa en la integración de información sensorial, vestibular, visual, somatosensorial y de otras fuentes. Esta información permite el seguimiento del cuerpo en el espacio y en relación con otros objetos y seres de nuestro entorno. El seguimiento de nuestro cuerpo, a su vez, orienta nuestros movimientos. La experiencia consciente de nuestro cuerpo es generalmente congruente en todas las modalidades sensoriales, de manera que lo que vemos de nuestro cuerpo es también lo que sentimos desde las perspectivas somatosensorial y vestibular. Las sensaciones y percepciones asociadas con nuestro cuerpo en movimiento también se pueden inducir en nuestra imaginación, aunque la mayor parte del tiempo en una forma atenuada. Recordemos que fue de esta forma como se percataron los investigadores de los primates de la existencia de las células en espejo. Mirar al otro implica reproducir en una parte de nuestro cerebro los movimientos que vemos, las emociones que percibimos e interpretamos. La empatía tiene ahí su asiento ${ }^{46}$.

\section{Despersonalización y desrealización}

Ambas experiencias pueden coexistir, pero son diferentes desde el punto de vista fenomenológico. La despersonalización se relaciona con la sensación personal de uno mismo percibido como un ente externo, como en el caso de la percepción extracorpórea (OBE), y una pérdida del control corporal, que puede hacer que las personas se sientan como robots. La desrealización, por otra parte, es una experiencia vinculada con el entorno, una sensación de estar en medio de un sueño. Sin embargo, ambas experiencias están íntimamente relacionadas en cuanto que pueden ocurrir simultáneamente. Es frecuente que se observen en personas con epilepsia y migraña ${ }^{45}$.

\section{Desapropiación corporal completa}

En un sentido radical, nosotros estamos en este mundo a través de nuestros cuerpos. Marleu-Ponty $(1957)^{47}$ afirmaba: «Estamos en el mundo a través de nuestros cuerpos, en tanto que percibimos el mundo a través de ellos». En teoría, si esto es cierto, no podríamos brincar, puentear, saltar, por decirlo de alguna forma, el cuerpo. Aprendemos del mundo a través de nuestro cuerpo, del mundo del cual somos también parte y participantes. En este sentido, el cuerpo es percibido como el horizonte entre uno mismo y el mundo que nos rodea, porque el mundo no es algo abstracto ni tampoco extraño, ya que estamos familiarizados con él de una manera prerreflexiva. El mundo es, entonces, parte del campo del cuerpo. Así pues, 
no hay otra forma de estar en el mundo que a través del cuerpo ${ }^{43}$.

Entonces, en el caso de la desapropiación corporal completa, el cuerpo pasa a ser un puro objeto entre otros objetos. En estos casos el cuerpo ya no es un horizonte a través del cual se observa y pertenecemos al mundo. Al perder sus límites, en situaciones extremas, el cuerpo puede volverse un enemigo, si es que se percibe continuamente como un estado de sufrimiento crónico. Las personas en esta situación pueden mutilar su cuerpo o incluso llegar al suicidio para no tener que seguir presentando este tipo de experiencia extrema ${ }^{20,21}$.

Si aceptamos el punto de vista fenomenológico, en el que experimentamos el mundo desde nuestro propio cuerpo, entonces debemos aceptar que, si el mundo se percibe a través de los "lentes» del cuerpo y el medio ambiente es hostil y repercute en el cuerpo, éste se convierte en un verdadero enemigo. Al ser nuestro cuerpo el punto de referencia con respecto al mundo, y si el cuerpo es un enemigo, esto sucede simplemente por la condición de estar en el mundo ${ }^{48}$.

Algunas personas transexuales, principalmente de hombre a mujer (mujeres transexuales), tienen una gran disforia con sus genitales, no los soportan, los sienten como algo que no debe estar en ese sitio, una aberración que les molesta, y es frecuente la automutilación, sobre todo en las etapas de la pubertad, en las que no entienden cómo pueden tener un pene si son mujeres; y no sólo eso, sino que además aumenta de tamaño, junto con el resto de caracteres sexuales secundarios. Éste es un claro ejemplo de ver una parte del cuerpo como un enemigo ${ }^{49,50}$.

Ramachandran, et al. ${ }^{32}$ publicaron una hipótesis a este respecto en la que decían que sería predecible que las personas transexuales con reasignación quirúrgica de genitales no tuvieran el fenómeno del miembro fantasma, que sí se presenta, por ejemplo, en hombres que tienen remoción del pene por cáncer o eventos traumáticos. Sin embargo, hay que aclarar al respecto que no hay reportes de ello sobre mujeres transexuales y que de hecho no se hace una extirpación de pene, sino que éste se utiliza para la neovagina y el glande con el meato urinario se colocan en el sitio que correspondería al clítoris, mientras que con las bolsas escrotales se hacen los labios mayores de la neovulva ${ }^{51}$.

En reportes de algunos pacientes que asisten a la clínica DIVERGEN (UNAM) y que se han sometido a este procedimiento quirúrgico, no hay evidencia del fenómeno del miembro fantasma; de hecho, hay una pérdida de la sensibilidad en toda la zona en que se han construido los genitales femeninos, sensibilidad que se recupera en un lapso de dos a tres años. Esto, además del detalle de integridad corporal, es importante porque algunas personas transexuales que no son instruidas respecto al cuidado de su neovagina tienden a fistularse hacia el recto o vejiga, pues al tener la zona parcialmente desensibilizada no perciben el dolor adecuadamente.

Un grupo significativo de mujeres transexuales presentan una negación de la existencia de su pene, y llegan a experimentar negligencia hacia el aseo y cuidados del mismo, con el pensamiento mágico de que algún día, al despertar, éste desaparecerá52.

Algunas de las preguntas a resolver en estas alteraciones son: ¿es posible detectar marcadoras de vulnerabilidad?, ¿se pueden hacer algunos procedimientos de tipo de las neuroprótesis para limitar las pérdidas de conectividad funcional de extremidades afectadas?, ¿es posible la neuromodulación en áreas como el tálamo para los dolores intratables? En víctimas de torturas crónicas, ¿qué procedimientos de neurorrehabilitación son los más eficaces? En nuestro país estamos en una situación de "guerra de baja intensidad", y la cantidad de personas sometidas a algunas de las situaciones descritas no es poca. En consecuencia, los médicos tenemos la responsabilidad de saber qué hacer para aliviar el daño, pero también si es posible prevenirlo y rehabilitarlo.

\section{Conclusiones}

Estamos contenidos dentro de nuestro cerebro, por lo menos en lo que respecta a la autoimagen corporal, y además tenemos una autoimagen distorsionada. Esto se genera en la región de asociación en los lóbulos parietal, temporal y occipital derechos como un constructo de nuestro desarrollo ontogénico y cultural, en donde nuestra neurobiología es la parte central de ello. Ahora sabemos que, en situaciones extremas de dolor, muerte inminente, tortura crónica, dolor por enfermedades e incluso sufrimiento por muertes de seres queridos, el sistema nervioso humano reacciona con una condición de aparente abandono del cuerpo.

\section{Bibliografía}

1. Ataria Y. Acute peritraumatic dissociation: in favor of a phenomenological inquiry. J Trauma Dissociation. 2014;15(3):332-47.

2. Metzinger $T$. The myth of cognitive agency: subpersonal thinking as a cyclically recurring loss of mental autonomy. Front Psychol. 2013;4:931.

3. Salomon R, Noel JP, Lukowska M, Faivre N, Metzinger T, Serino A et al. Unconscious integration of multisensory bodily inputs in the peripersonal space shapes bodily self-consciousness. Cognition. 2017; 166:174-83. 
4. Metzinger T. Why are out-of-body experiences interesting for philosophers? The theoretical relevance of OBE research. Cortex. 2009; 45(2):256-8.

5. Elias GA, Bieszczad KM, Weinberger NM. Learning strategy refinement reverses early sensory cortical map expansion but not behavior: Support for a theory of directed cortical substrates of learning and memory. Neurobiol Learn Mem. 2015;126:39-55.

6. Blanke O, Mohr C, Michel CM, Pascual-Leone A, Brugger P, Seeck M, et al. Linking out-of-body experience and self processing to mental ownbody imagery at the temporoparietal junction. J Neurosci. 2005;25(3):550-7.

7. Giummarra MJ, Gibson SJ, Georgiou-Karistianis N, Bradshaw JL. Central mechanisms in phantom limb perception: the past, present and future. Brain Res Rev. 2007;54(1):219-32.

8. Giummarra MJ, Gibson SJ, Georgiou-Karistianis N, Bradshaw JL. Mechanisms underlying embodiment, disembodiment and loss of embodiment. Neurosci Biobehav Rev. 2008;32(1):143-60.

9. Nickerson A, Garber B, Ahmed O, Asnaani A, Cheung J, Hofmann SG et al. Emotional suppression in torture survivors: Relationship to posttraumatic stress symptoms and trauma-related negative affect. Psychiatry Res. 2016;242:233-9.

10. Tsur N, Defrin R, Ginzburg K. Posttraumatic stress disorder, orientation to pain, and pain perception in ex-prisoners of war who underwent torture. Psychosom Med. 2017;79(6):655-63.

11. Tsur N, Shahar G, Defrin R, Lahav Y, Ginzburg K. Torturing personification of chronic pain among torture survivors. J Psychosom Res. 2017; 99:155-61

12. Weinberg M, Besser A, Ataria $Y$, Neria $Y$. Survivor-spouse dissociation and posttraumatic stress disorder: Personal and dyad relationships. J Trauma Dissociation. 2016;17(4):448-59.

13. Williams ACC, Amris K. Treatment of persistent pain from torture: review and commentary. Med Confl Surviv. 2017;33(1):60-81.

14. Ataria $Y$. When the body becomes the enemy: Disownership toward the body. Philosophy, Psychiatry and Psychology. 2016;23(1):15.

15. Radziwillowicz W, Lewandowska M. From traumatic events and dissociation to body image and depression symptoms - in search of self-destruction syndrome in adolescents who engage in nonsuicidal self-injury. Psychiatr Pol. 2017;51(2):283-301.

16. Baars EW, van der Hart O, Nijenhuis ER, Chu JA, Glas G, Draijer N Predicting stabilizing treatment outcomes for complex posttraumatic stress disorder and dissociative identity disorder: an expertise-based prognostic model. J Trauma Dissociation. 2011;12(1):67-87.

17. Soffer $Y$, Wolf JJ, Ben-Ezra M. Correlations between psychosocial factors and psychological trauma symptoms among rescue personnel. Prehosp Disaster Med. 2011;26(3):166-9.

18. Kutcher S, Szumilas M. Distinguishing suicide attempts from nonsuicidal self-harming behaviors. J Am Acad Child Adolesc Psychiatry. 2009; 48(10):1039; author reply 1039-41.

19. Schechter M, Lineberry TW. Self-harming behavior and suicidality: suicide risk assessment. Suicide Life Threat Behav. 2011:41(2):227-34

20. Liljedahl SI, Helleman M, Daukantaite D, Westrin A, Westling S. A standardized crisis management model for self-harming and suicidal individuals with three or more diagnostic criteria of borderline personality disorder: The Brief Admission Skane randomized controlled trial protocol (BASRCT). BMC Psychiatry. 2017;17(1):220.

21. Mansfield AJ, Bender RH, Hourani LL, Larson GE. Suicidal or self-harming ideation in military personnel transitioning to civilian life. Suicide Life Threat Behav. 2011;41(4):392-405.

22. Marzano L, Ciclitira K, Adler J. The impact of prison staff responses on self-harming behaviours: prisoners' perspectives. Br J Clin Psychol. 2012; 51(1):4-18.

23. McDaid D, Tsiachristas A, Hawton K. Understanding the true economic impact of self-harming behaviour - Authors' reply. Lancet Psychiatry. 2017;4(12):901.

24. Tormoen AJ, Rossow I, Mork E, Mehlum L. Contact with child and adolescent psychiatric services among self-harming and suicidal adolescents in the general population: a cross sectional study. Child Adolesc Psychiatry Ment Health. 2014;8:13.
25. Tormoen AJ, Groholt B, Haga E, Brager-Larsen A, Miller A, Walby F et al. Feasibility of dialectical behavior therapy with suicidal and self-harming adolescents with multi-problems: training, adherence, and retention. Arch Suicide Res. 2014:18(4):432-44.

26. Thelle H. Torture in China. Torture. 2006;16(3):268-75.

27. Wang ZR. [Review on experimental studies on Yin-yang theory of traditional Chinese medicine]. Zhonghua Yi Shi Za Zhi. 2005;35(1):22-4.

28. Association AP. Diagnostic and Statistical Manual of Mental Disorders. Vol. 1. USA: American Psychiatric Association; 2013

29. Larsen SE, Berenbaum H. Did the DSM-5 Improve the Traumatic Stressor Criterion?: Association of DSM-IV and DSM-5 Criterion A with Posttraumatic Stress Disorder Symptoms. Psychopathology. 2017;50(6):373-8.

30. Buklina SB. [Pseudopolymelia and disorders of body scheme]. Zh Nevrol Psikhiatr Im S S Korsakova. 2008;108(1):4-11.

31. Franz EA, Fu Y, Moore M, Winter T, Mayne T, Debnath R, et al. Fooling the brain by mirroring the hand: Brain correlates of the perceptual capture of limb ownership. Restor Neurol Neurosci. 2016;34(5):721-32.

32. Ramachandran VS. Consciousness and body image: lessons from phantom limbs, Capgras syndrome and pain asymbolia. Philos Trans R Soc Lond B Biol Sci. 1998;353(1377):1851-9.

33. López-lbor JJ, Ortiz T, López-lbor MI. Perception, experience and body identity. Actas Esp Psiquiatr. 2011;39 Suppl 3:3-118.

34. First MB, Fisher CE. Body integrity identity disorder: the persistent desire to acquire a physical disability. Psychopathology. 2012;45(1):3-14.

35. Carinci AJ, Mehta P, Christo PJ. Chronic pain in torture victims. Curr Pain Headache Rep. 2010;14(2):73-9.

36. Rasmussen A, Keatley E, Paz C. Disengagement in a torture treatment program. Psychiatr Serv. 2013;64(12):1280.

37. Nortvedt F, Engelsrud G. "Imprisoned" in pain: analyzing personal experiences of phantom pain. Med Health Care Philos. 2014;17(4):599-608.

38. Blanke $O$, Landis $T$, Spinelli L, Seeck M. Out-of-body experience and autoscopy of neurological origin. Brain. 2004;127(Pt 2):243-58.

39. Bunning S, Blanke $\mathrm{O}$. The out-of body experience: precipitating factors and neural correlates. Prog Brain Res. 2005;150:331-50.

40. Salin-Pascual RJ. La autopercepción en el caso de la parálisis del sueño: Un estado de conciencia dentro del sueño MOR. Rev Mex Neurosci. 2016;17(6):14

41. Bourdin P, Barberia I, Oliva R, Slater M. A virtual out-of-body experience reduces fear of death. PLoS One. 2017;12(1):e0169343.

42. Bokkon I, Mallick BN, Tuszynski JA. Near death experiences: a multidisciplinary hypothesis. Front Hum Neurosci. 2013;7:533.

43. Jalal B, Ramachandran VS. Sleep Paralysis, "The Ghostly Bedroom Intruder" and Out-of-Body Experiences: The Role of Mirror Neurons. Front Hum Neurosci. 2017;11:92

44. Nelson KR. Near-death experience: arising from the borderlands of consciousness in crisis. Ann N Y Acad Sci. 2014;1330:111-9.

45. Smith AM, Messier C. Voluntary Out-of-Body Experience: An fMRI Study. Front Hum Neurosci. 2014;8:70.

46. Smetacek V. Recalibrating sleep: is recalibration and readjustment of sense organs and brain-body connections the core function of sleep? Med Hypotheses. 2010;75(4):401-4.

47. Marleu-Ponty M. Fenomenología de la percepción. Vol. 1. México: Fondo de Cultura Económico; 1957.

48. Quigley J, Rasmussen S, McAlaney J. The Social Norms of Suicidal and Self-Harming Behaviours in Scottish Adolescents. Int $\mathrm{J}$ Environ Res Public Health. 2017:14(3).

49. McDermott E. Asking for help online: lesbian, gay, bisexual and trans youth, self-harm and articulating the 'failed' self. Health (London). 2015; 19(6):561-77.

50. Roen K. The body as a site of gender-related distress: ethical considerations for gender variant youth in clinical settings. J Homosex. 2016;63(3):306-22.

51. Case LK, Brang D, Landazuri R, Viswanathan P, Ramachandran VS. Altered White Matter and Sensory Response to Bodily Sensation in Female-to-Male Transgender Individuals. Arch Sex Behav. 2017;46(5):1223-37.

52. Namba $Y$, Sugiyama N, Yamashita S, Tokuyama E, Hasegawa K, Kimata $Y$. Phantom erectile penis after sex reassignment surgery. Acta Med Okayama. 2008;62(3):213-6. 